\title{
CAPACITIVE TRANSDUCER ENHANCEMENT ON QUADRATURE COMPENSATION ELECTRODE OF YAW RATE GYROSCOPE
}

\author{
Peng Shao, Eduardo Canales, and Peiqing Zhu \\ NXP Semiconductors, Chandler, Arizona, USA
}

\begin{abstract}
Being the major error source of Coriolis vibratory gyroscope, quadrature error is always well-compensated for high performance MEMS gyroscopes. This paper presents a novel design of quadrature compensation unit (QCU) electrodes for yaw rate gyroscope, with enhanced compensation efficiency. The design decreases the effective capacitance gap during half cycle of the drive oscillation. Theoretical analysis indicates that, these QCU electrodes will create a sinusoidal force that is inherently modulated by drive mode displacement, with about $3 \mathrm{X}$ increase in compensation efficiency. Both QCU designs are implemented on the same gyroscope test structure. Wafer level probe data is showing the predicted increase of QCU efficiency and assembled parts data is showing a reduced voltage needed for quadrature compensation.
\end{abstract}

\section{INTRODUCTION}

With the boost of automotive industry, there is a need of high performance MEMS gyroscopes with small offset drift and noise density, for the applications of Electronic Stability Control (ESC), Advanced Driver Assistance Systems (ADAS), and Autonomous Driving.

Quadrature error is a major error source of Coriolis vibratory gyroscope. It is mainly caused by variations of MEMS process technology, such as etching uniformity, side wall slope and is difficult to predict. Large quadrature error forces the analog front-end to have wide operating range which decreases the noise and power performance [1]. It also tends to shift with temperature and causes large temperature coefficient of offset (TCO) and temperature coefficient of sensitivity (TCS). For high performance gyroscopes, quadrature error needs to be compensated by dedicated electrodes with a closed loop control system. The controller reads out the demodulated quadrature signal from sense electrode as an error signal and tries to null it with compensation voltages.

The most commonly used method of nulling quadrature error is to apply a DC voltage on Quadrature Compensation Unit (QCU), which is placed on the portion of mass that has both drive mode and sense mode motion at the same time [2]-[4]. The QCU consists of interdigitated fingers, whose gaps are defined by the minimum trench width of the process technology. In this paper, a novel QCU structure design with capacitive transducer enhancement is introduced. The design reduces the effective capacitance gap during half period of the drive oscillation. By placing QCU in pairs, compensation force will be magnified by 3 times [5].

This paper first describes the working principle of quadrature compensation and its conventional design, then propose a method of improving its compensation efficiency. Two types of design are built into the same gyroscope test structure. Wafer probe data and assembled parts data are shown to prove the theory.

\section{THEORY}

\section{Conventional Design (Type A)}

Quadrature error originates from structural non-ideality of drive oscillator. Drive suspensions are design as such that they are flexible in drive direction and rigid in sense direction. However, the

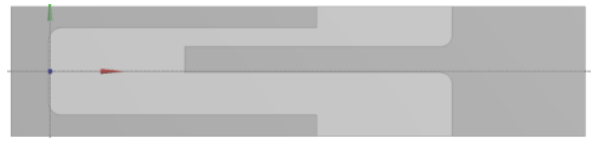

Figure 1: Conventional Quadrature Compensation Unit (Type A) Structure Design with biased electrostatic gap

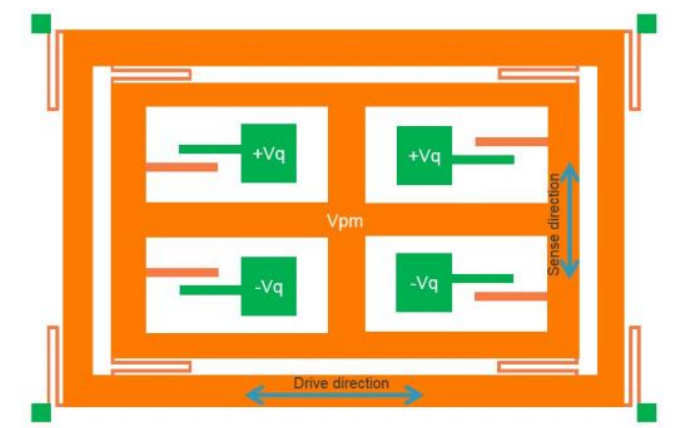

Figure 2: Configuration of Four Groups of $Q C U$ fingers

stiffness of drive suspension in sense direction is finite, and will create coupling between drive and sense mode. Moreover, manufacturing imperfection will couple drive mode into sense mode as well. Consider a lumped parameter model of a vibratory gyroscope:

$$
\left[\begin{array}{cc}
m_{d} & 0 \\
0 & m_{s}
\end{array}\right]\left[\begin{array}{c}
\ddot{x} \\
\ddot{y}
\end{array}\right]+\left[\begin{array}{cc}
c_{x} & 0 \\
0 & c_{y}
\end{array}\right]\left[\begin{array}{l}
\dot{x} \\
\dot{y}
\end{array}\right]+\left[\begin{array}{cc}
k_{x} & k_{x y} \\
k_{y x} & k_{y}
\end{array}\right]\left[\begin{array}{l}
x \\
y
\end{array}\right]=\left[\begin{array}{c}
F_{d} \\
-2 m_{C} \Omega_{z} \dot{x}
\end{array}\right]
$$

Where $m_{d}$ and $m_{s}$ are drive mass and sense mass, respectively, $F_{d}$ is driving force, the term $-2 m_{c} \Omega_{z} \dot{x}$ represents Coriolis force from yaw rate, $k_{x y}$ and $k_{y x}$ are two stiffness cross-coupling term, in which $k_{y x}$ stands for the coupling from drive axis (x) to sense axis (y). The term $k_{y x} x$ can be defined as quadrature force, which is a time varying force in phase with drive displacement. To cancel this quadrature force, a compensation force in phase with drive displacement is required.

In order to generate a compensation force in phase with drive displacement, interdigitated fingers with bias gaps are used as figure 1 shows. The overlapping of movable and stationary fingers is larger than the travel range of movable mass. Figure 2 shows the configuration of four groups of QCU fingers placed inside Coriolis mass of a single mass gyroscope. The top two groups have the same electric potential of $+\mathrm{V}_{\mathrm{q}}$, while the bottom two groups have the electric potential of $-\mathrm{V}_{\mathrm{q}}$. Movable mass is biased at $\mathrm{V}_{\mathrm{pm}}$. The quadrature compensation force of the upper left group is,

$$
F_{q c_{-} u l}=-\frac{\partial E}{\partial y}=\frac{1}{2} N \varepsilon h \frac{x_{0}+x \sin \omega_{d} t}{y_{0}{ }^{2}}\left(V_{p m}-V_{q}\right)^{2}
$$

Where $\mathrm{x}_{0}$ is the stationary overlapping length, $\mathrm{N}$ is the number of fingers, $\mathrm{x}$ is the drive direction, $\mathrm{y}$ is the sense direction, $\mathrm{h}$ is the structure layer thickness, $\omega_{\mathrm{d}}$ is drive resonator frequency.

Considering the 180 degree phase difference and opposite in direction of right finger, and the $-\mathrm{Vq}$ bias for the bottom groups of 
fingers, the quadrature compensation force of the upper right group is,

$$
F_{q c_{-} u r}=-\frac{1}{2} N \varepsilon h \frac{x_{0}-x \sin \omega_{d} t}{y_{0}{ }^{2}}\left(V_{p m}-V_{q}\right)^{2}
$$

The total force from upper two groups that biased at $+\mathrm{V}_{\mathrm{q}}$ is,

$F_{q c_{-} u a}=N \varepsilon h \frac{x \sin \omega_{d} t}{y_{0}{ }^{2}}\left(V_{p m}-V_{q}\right)^{2}$

Similarly, the total force from lower two groups is,

$$
F_{q c_{-} l a}=-N \varepsilon h \frac{x \sin \omega_{d} t}{y_{0}{ }^{2}}\left(V_{p m}+V_{q}\right)^{2}
$$

By adding the forces from upper group and lower group together, the total quadrature compensation force will be,

$$
F_{q c}=4 N \varepsilon h \frac{V_{p m} V_{q}}{y_{0}{ }^{2}} x \sin \omega_{d} t
$$

The total compensation force is proportional to number of fingers, layer thickness, proof mass voltage, quadrature voltage and drive amplitude, inversely proportional to the square of gap size, which is a limiting factor defined by the design rule of the process technology.

\section{Novel Design (Type B)}

The novel design proposed in this paper introduces a way of enhancing the capacitance as illustrated in Figure 3. The enhanced structure modifies both movable and fixed fingers, reducing the effective capacitive gap during the positive half cycle, while the

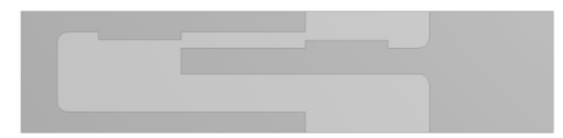

Figure 3: Proposed Quadrature Compensation Unit (Type B) Structure Design with enhancement structure

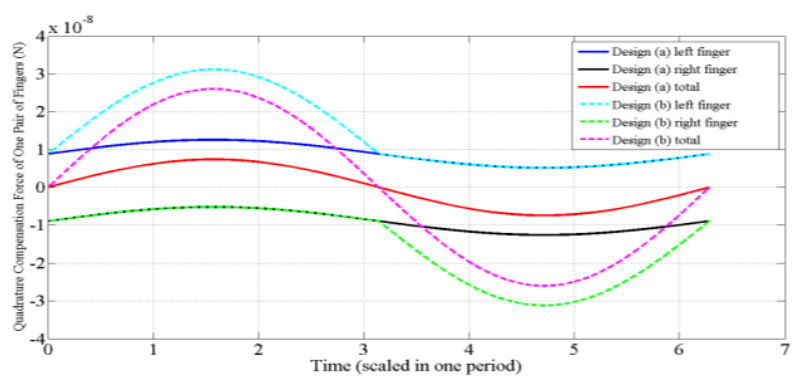

Figure 4: Comparison of both QCU finger design. The blue and black solid line is the force of left finger and right finger of $Q C U$ design type A. They added together (red solid line) to be the total force of design type $A$. The dotted blue line and dotted green line is the force of left finger and right finer of $Q C U$ design type $B$, who added together (dotted pink line) to be the total force of new finger design. (dimensions: initial overlap $=12 \mathrm{um}$, drive amplitude $=5 \mathrm{um}$, structure thickness $=23.5 \mathrm{um}$, nominal gap $=$ $1.5 \mathrm{um}$, reduced gap during travel $=0.7 \mathrm{um}$

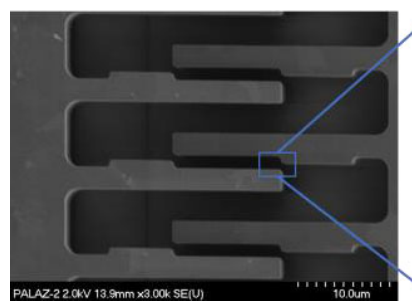

(a)

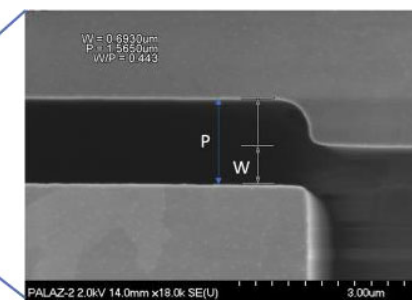

(b)
Figure 5: SEM image of (a) one group of QCU fingers; (b) Zoomed-in view and detailed dimension measurement fingers on the other side reduces the gap during the negative half cycle. The combination of two groups of fingers create a sinusoidal force with larger amplitude and is in phase with drive amplitude.

Even though at the edge of the added block, the gap is smaller than the minimum gap, it will not affect manufacturability since the overlapping area is negligible. DRIE can define narrower capacitive gap with small overlapping area, compared with long trenches for springs and interdigitated fingers.

Figure 4 shows the compensation force of both designs in one cycle. The blue and black solid line is the force of left finger and right finger of QCU design type A (conventional finger design). They added together (red solid line) to be the total force of design type A. The dotted blue line and dotted green line is the force of left finger and right finger of QCU design type B (novel finger design), who added together (dotted pink line) to be the total force of design type B. It can be seen from figure 4, design type B is providing 3 times force design type A can provide, while keeping a sinusoidal compensation force. The dimensions in this calculation are numbers from process technology and MEMS design, and listed in Figure 4.

\section{EXPERIMENT}

\section{Wafer Level Probe}

To verify the increase of efficiency of QCU design, both designs were implemented on the same MEMS gyroscope design respectively, and were fabricated using the same process technology reported in [6]. The process technology is capable of defining parallel plate capacitor with minimum gap of $1.5 \mathrm{um}$. For short trenches whose length is smaller than $2 \mathrm{um}$, trenches with $0.7 \mathrm{um}$ in width can be etched. These trenches are usually used to define lateral shock stops. Figure 5 shows the SEM image of the improved design, (a) is a set of QCU fingers and (b) is zoomed-in view of detailed structure. The measurement of the SEM image shows a nominal gap of $1.565 \mathrm{um}$, and enhanced gap of $0.673 \mathrm{um}$.

The MEMS wafer was probed using lock-in amplifier as illustrated in figure 6. Lock-in amplifier actuate drive resonator from Drive Actuation Unit (DAU), and acquire drive motion signal from Drive Measurement Unit (DMU), and quadrature signal from Sense Measurement Unit (SMU) at the same time. Both magnitude and phase value need to be read for correct polarity of quadrature signal. The current drawn from DMU is calculated as:

$$
i_{d r v}=N_{D M U} \frac{\varepsilon_{0} H V}{d} \frac{\partial x}{\partial t}=N_{D M U} \frac{\varepsilon_{0} H V x_{d} \omega_{d} \cos \omega_{d} t}{d}
$$

Where, $\mathrm{N}_{\mathrm{DMU}}$ is the number of capacitors of DMU fingers, $\mathrm{H}$ is the silicon layer thickness, $\mathrm{V}$ is the biased voltage on proof mass, $\mathrm{d}$ is the capacitance gap size of DMU and SMU, $\mathrm{x}_{\mathrm{d}}$ is the drive resonator amplitude, $\omega_{\mathrm{d}}$ is the drive resonator angular frequency.

The current drawn from SMU is calculated as:

$i_{\text {quad }}=-N_{S M U} \frac{\varepsilon_{0} L H V}{d^{2}} \frac{\partial y}{\partial t}=-N_{S M U} \frac{\varepsilon_{0} L H V y_{q} \omega_{d} \cos \left(\omega_{d} t\right)}{d^{2}}$

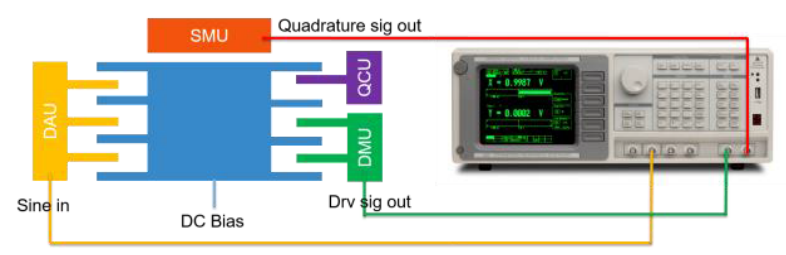

Figure 6: Schematic of wafer level quadrature probing. DAU: Drive Actuation Unit. DMU: Drive Measurement Unit. SMU: Sense Measurement Unit. QCU: Quadrature Compensation Unit. 
Where, $\mathrm{N}_{\mathrm{SMU}}$ is the number of capacitors of SMU, $\mathrm{y}_{\mathrm{q}}$ is the displacement on sense axis as a result of quadrature, $\mathrm{L}$ is the SMU length.

By taking the ratio between drive signal and quadrature signal, the ratio between drive displacement amplitude and quadrature displacement amplitude can be calculated as:

$$
\frac{y_{q}}{x_{d}}=\frac{\left|i_{q u a d}\right|}{\left|i_{d r v}\right|} \cdot \frac{N_{D M U} \cdot d}{N_{S M U} \cdot L}
$$

The quadrature displacement amplitude then can be scaled with rate sensitivity for the quadrature level in degree per second (dps). Consider a Coriolis induced displacement,

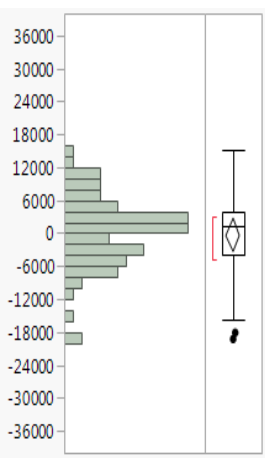

(a)

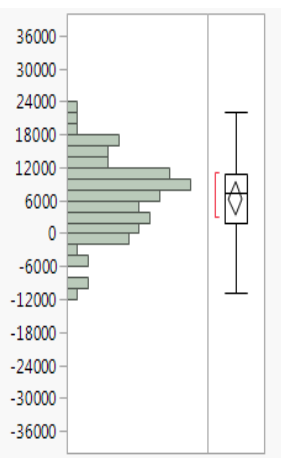

(b)
Figure 7: Quadrature level (in dps) measured from $Q C U$ design type $A$, (a) without no QCU differential voltage; (b) with 1 VDC differential voltage

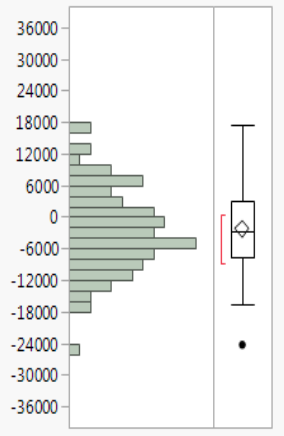

(a)

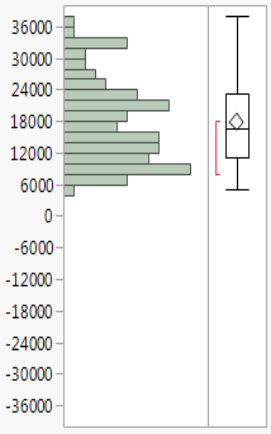

(b)
Figure 8: Quadrature level (in dps) measured from $Q C U$ design type $B$, (a) without no $Q C U$ differential voltage; (b) with 1 VDC differential voltage

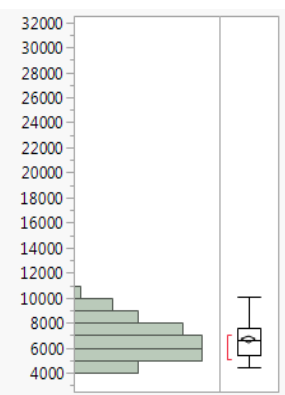

(b)

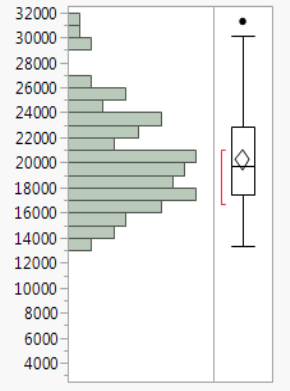

(b)
Figure 9: Quadrature compensation efficiency (dps/V) of (a) $Q C U$ design type $A$; (b) $Q C U$ design type $B$, demonstrating higher efficiency type $B Q C U$ design

$$
y_{\text {cor }}=\frac{F_{\text {cor }} Q_{\text {eff }}}{k_{y}}=\frac{2 m_{s} \Omega x_{d} \omega_{d} Q_{\text {eff }}}{m_{s} \omega_{s}^{2}}=\frac{2 \Omega x_{d} \omega_{d} Q_{\text {eff }}}{\omega_{s}^{2}}
$$

Where, $\mathrm{m}_{\mathrm{s}}$ is the mass of sense resonator, $\Omega$ is the angular rate, $\mathrm{Q}_{\text {eff }}$ is the effective quality factor considering the frequency spacing between drive and sense mode, $\omega_{s}$ is the angular frequency of sense resonator.

$$
\begin{aligned}
& \text { Quadrature signal can be scaled as: } \\
& \Omega=\frac{\left|i_{\text {quad }}\right|}{\left|i_{\text {drv }}\right|} \cdot \frac{N_{D M U} \cdot d}{N_{S M U} \cdot L} \cdot \frac{\omega_{s}{ }^{2}}{2 \omega_{d} Q_{e f f}}
\end{aligned}
$$

To compare the QCU compensation efficiency, the above procedure was repeated with $1 \mathrm{~V}$ QCU differential voltage (+1VDC on QCU+, -1VDC on QCU-). The difference of the quadrature level between the two measurement is the QCU efficiency in terms of $\mathrm{dps} / \mathrm{V}$. Designs with both types of QCU are evaluated as described above.

Figure 7(a) and figure 8(a) demonstrate the distribution of quadrature level without QCU differential voltage for design type A and design type $\mathrm{B}$, respectively. Their average values are both close to $0 \mathrm{dps}$ as expected, and go from $-18000 \mathrm{dps}$ to $+18000 \mathrm{dps}$. Figure 7(b) and figure 8(b) shows the quadrature level with $1 \mathrm{~V}$ QCU voltage for design type $\mathrm{A}$ and design type $\mathrm{B}$. As the figures are showing, design type $\mathrm{B}$ is shifting the quadrature level much more than design type A. Figure 9 plots the QCU compensation efficiency of both designs. Type A design has the mean value of $6700 \mathrm{dps} / \mathrm{V}$, with variation from $4000 \mathrm{dps} / \mathrm{V}$ to $11000 \mathrm{dps} / \mathrm{V}$. Type B design has the mean value of $20200 \mathrm{dps} / \mathrm{V}$, and varies from $13000 \mathrm{dps} / \mathrm{V}$ to $37000 \mathrm{dps} / \mathrm{V}$, due to the process variation of MEMS technology. This demonstrates $3 \mathrm{X}$ higher efficiency of type B compared with type A.

Quadrature efficiency is mapped and plotted on wafer scale to see the trend within single wafer. Two type of designs are on two different wafers. In general, QCU efficiency goes from high to low from northeast to southwest of the wafer. Some minor deviation is due to wafer-to-wafer variation.

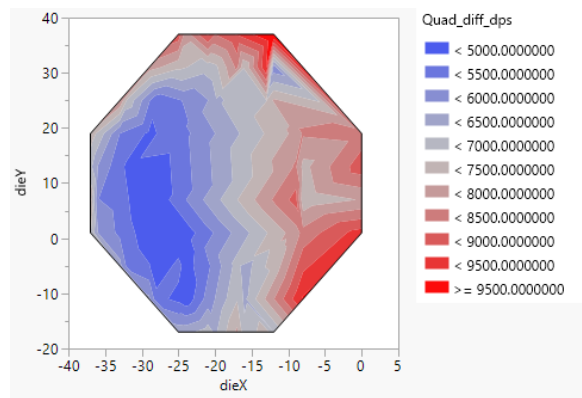

(a)

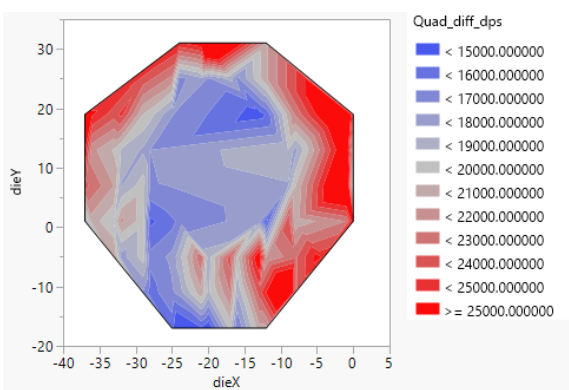

(b)

Figure 10: Wafer map of QCU efficiency. (a) Wafer of type A design; (b) Wafer of type $B$ design 


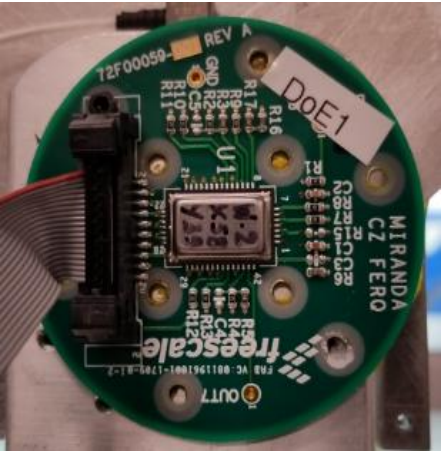

Figure 11:Board-mounted in-frame package for evaluation of $Q C U$ differential voltages of engineering samples.

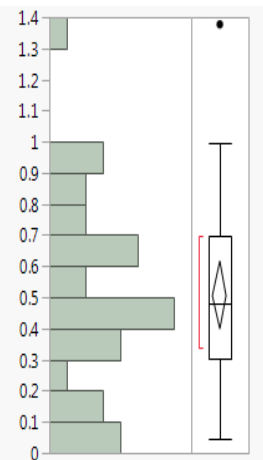

(a)

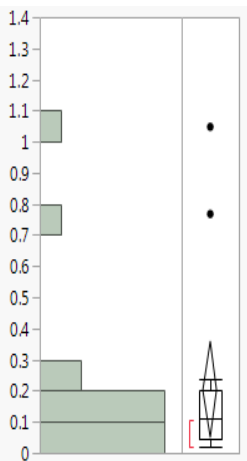

(b)
Figure 12: QCU differential voltage to cancel quadrature error (a) $Q C U$ design type A; (b) QCU design type B.

\section{Assembled Part Characterization}

MEMS dies are built with ASIC dies that can provide QCU voltage with a closed loop control system. The board-mounted inframe premolded package is shown in figure 11. Quadrature loop is trimmed to extract QCU voltages used to null quadrature error, shown in figure 12(a) and (b) for type A and type B design, respectively. The QCU driver has certain limit on the voltage that can be provided, leaving some type A parts not trimmable (not included in figure 12(a)).

\section{SUMMARY}

This paper proposed a novel quadrature compensation electrode design to improve its efficiency, thus power and/or die area can be saved. Analytical calculation predicts $3 \mathrm{X}$ increase in efficiency. Both QCU designs are implemented on the same gyroscope test structure. Both wafer level probe and assembled part evaluation demonstrate the increase in efficiency that agrees with the analysis. Table 1 is summarizing the mean value for all the measurement done on both designs.

Table 1. Summary of measured mean value

\begin{tabular}{|c|c|c|c|c|}
\hline $\begin{array}{l}\text { QCU } \\
\text { Design }\end{array}$ & $\begin{array}{c}\text { Quad. @ } \\
\text { 0V (dps) }\end{array}$ & $\begin{array}{c}\text { Quad. @ } \\
\text { 1V (dps) }\end{array}$ & $\begin{array}{c}\text { QCU Efficiency } \\
\text { (dps/V) }\end{array}$ & $\begin{array}{c}\text { QCU } \\
\text { voltage (V) }\end{array}$ \\
\hline A & -397 & 6273 & 6671 & 0.5 \\
\hline B & -2307 & 17902 & 20209 & 0.2 \\
\hline
\end{tabular}

\section{REFERENCES}

[1] C. Acar and A. Shkel, MEMS Vibratory Gyroscopes. 2009.

[2] M. A. Lemkin, W. A. Clark, T. N. Juneau, and A. W. Roessig, "Structure for attenuation or cancellation of quadrature error," US7051590 B1, 30-May-2006.

[3] E. Tatar, S. E. Alper, and T. Akin, "Quadrature-Error Compensation and Corresponding Effects on the Performance of Fully Decoupled MEMS Gyroscopes," J. Microelectromechanical Syst., vol. 21, no. 3, pp. 656-667, Jun. 2012.

[4] M. F. Zaman, A. Sharma, Z. Hao, and F. Ayazi, "A ModeMatched Silicon-Yaw Tuning-Fork Gyroscope With Subdegree-

Per-Hour Allan Deviation Bias Instability," J. Microelectromechanical Syst., vol. 17, no. 6, pp. 1526-1536, Dec. 2008.

[5] P. Shao, "Mems device with capacitance enhancement on quadrature compensation electrode," US20170138734 A1, 18-May2017.

[6] A. D. Oliver, Y. L. Teo, A. Geisberger, R. F. Steimle, T. Cassagnes, K. Adhikari, D. Sadler, and A. McNeil, "A new three axis low power MEMS gyroscope for consumer and industrial applications," in 2015 Transducers - 2015 18th International Conference on Solid-State Sensors, Actuators and Microsystems (TRANSDUCERS), 2015, pp. 31-34.

\section{CONTACT}

*P. Shao, tel: +1-480-814-2958; P.Shao@nxp.com 